\title{
On-Line Covering a Cube by a Sequence of Cubes
}

\author{
W. Kuperberg \\ Department of Mathematics, Auburn University, \\ Auburn, AL 36849-5310, USA \\ wkuperb@ducvax.auburn.edu
}

\begin{abstract}
A procedure for packing or covering a given convex body $K$ with a sequence of convex bodies $\left\{C_{i}\right\}$ is an on-line method if the sets $C_{i}$ are given in sequence, and $C_{i+1}$ is presented only after $C_{i}$ has been put in place, without the option of changing the placement afterward. The "one-line" idea was introduced by Lassak and Zhang [6] who found an asymptotic volume bound for the problem of on-line packing a cube with a sequence of convex bodies. In this note a problem of Lassak is solved, concerning on-line covering a cube with a sequence of cubes, by proving that every sequence of cubes in the Euclidean space $E^{d}$ whose total volume is greater than $4^{d}$ admits an on-line covering of the unit cube. Without the "on-line" restriction, the best possible volume bound is known to be $2^{d}-1$, obtained by Groemer [2] and, independently, by Bezdek and Bezdek [1]. The on-line covering method described in this note is based on a suitable cube-filling Peano curve.
\end{abstract}

\section{Introduction}

A general problem on covering or packing a convex set with a sequence of convex sets reads as follows (see [3]): If $K$ is a convex subset of the $d$-dimensional Euclidean space $E^{d}$, and if $\left\{C_{i}\right\}$ is a finite or infinite sequence of closed convex subsets of $E^{d}$, is it possible to apply to the sets $C_{i}$ rigid motions (or perhaps only translations) so that the resulting sets cover $K$ or are contained in $K$ and have mutually disjoint interiors? Furthermore, if such a covering or packing exists, the problem of estimating the best possible efficiency arises. Groemer [3] surveys a multitude of results on these questions, most of which have been obtained in recent decades.

Lassak and Zhang [6] add an interesting twist to the problem: Suppose that as the sets $C_{i}$ are given in sequence, one at a time, each of them must be assigned its place before the next one appears, and that the placement cannot be modified 
afterward. After Lassak and Zhang, a packing or covering process under such a severe but natural restriction, is called an on-line packing or an on-line covering. They consider the problem for packings only, and obtain an asymptotically "best possible" result, leaving the on-line covering problem open.

At the 1991 Discrete Mathematics Conference in Dortmund, Germany, Lassak asked the following, specific question: Does every sequence of sequares (in $E^{2}$ ) whose sum of areas is infinite admit an on-line covering of the unit square? In response to this question, we present the following:

Theorem. Every sequence of cubes in $E^{d}$ whose sum of volumes is greater than $4^{d}$ admits an on-line covering of the unit cube.

We do not know whether the number $4^{d}$ represents the best possible efficiency estimate; we suspect it does not, but we do know that such an estimate cannot fall below $2^{d}-1$, which is the best possible for the "usual" problem (i.e., without the on-line restriction) (see [2] or [1]). Also, the problem of generalizations of the above theorem remains open. Specifically, does every sequence of rectangular boxes in $E^{d}$ with a bounded set of diameters and of infinite total volume admit an on-line [translational] covering of the unit cube? Observe that the assumption of a bounded set of diameters is essential: without it the question is trivially answered in the negative.

In Section 2 we describe how a cube-filling Peano curve can yield an on-line method for covering the cube with a sequence of sets. Section 3 contains the construction of a Peano curve particularly suitable for that purpose, and in Section 4 we prove the main result.

\section{On-Line Covering a Cube Along a Peano Curve}

Let $\varphi$ be a continuous function mapping the interval $[0,1]$ onto the unit cube in $E^{d}$, a cube-filling Peano curve for short. We associate with the curve an on-line method of [translative] covering of the unit cube with a (finite or infinite) sequence of closed sets $\left\{C_{i}\right\}$ with nonempty interiors as follows. Let $a_{1}$ be the maximum number in $[0,1]$ such that the image $\varphi\left(\left[0, a_{1}\right]\right)$ can be covered with a congruent replica [a translate] of $C_{1}$. Place [translate] $C_{1}$ so that it covers $\varphi\left(\left[0, a_{1}\right]\right)$. Next, let $a_{2}$ be the maximum number in $\left[a_{1}, 1\right]$ such that the image $\varphi\left(\left[a_{1}, a_{2}\right]\right)$ can be covered with a congruent replica [a translate] of $C_{2}$. Place [translate] $C_{2}$ so that it covers $\varphi\left(\left[a_{1}, a_{2}\right]\right)$ (see Fig. 1).

Continue defining the numbers $a_{i}$ and the corresponding placements [translations] of the sets $C_{i}$ in this manner. If at any time the value of $a_{i}$ reaches 1 , then the procedure stops having accomplished its purpose: the unit cube has been covered by (a finite number of) the $C_{i}$ 's. On the other hand, if $a_{i}<1$ for all $i$, the procedure fails.

Obviously, the success of the method depends on the curve $\varphi$ and on the properties of the sequence $\left\{C_{i}\right\}$. We illustrate the method with two examples of on-line covering problems in the plane. 


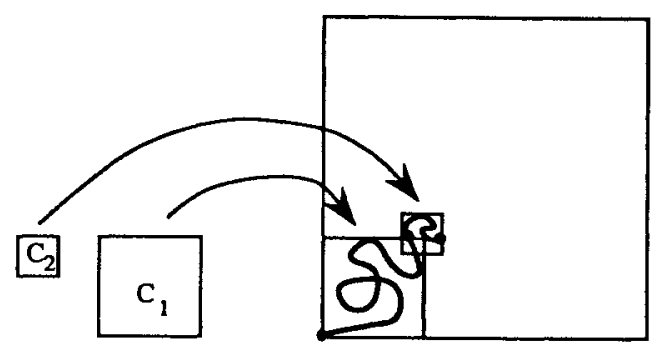

Fig. 1. The positions of $C_{1}$ and $C_{2}$.

Example 1. For the purpose of on-line covering th unit square with a sequence $\left\{C_{i}\right\}$ of squares, let us use the familiar, "standard" square-filling curve $\varphi$ whose description can be found in [5] (Fig. 2 shows three initial approximations of $\varphi$ ).

Observe that $\varphi$ maps each subinterval $\left[9^{-k},(j+1) 9^{-k}\right]$ of $[0,1]$ onto a square of area $9^{-k}$, the same as the length of the subinterval. Also, $\varphi$ maps two adjacent subintervals $\left[(j-1) 9^{-k}, j 9^{-k}\right]$ and $\left[j 9^{-k},(j+1) 9^{-k}\right]$ onto two squares that share an edge. It follows that the image of any subinterval of length $9^{-k}$ can be covered by a square of area $4 \times 9^{-k}$, which is four times the length of the subinterval. Consequently, the image of any subinterval $J$ can be covered by a square of area $9 \times 4=36$ times the length of $J$. It follows that in the on-line covering method described above, for each $i=2,3, \ldots$, the number $a_{i}-a_{i-1}$ is greater than or equal to $\frac{1}{36}$ times the area of $C_{i}$.

Thus, if the total area of the squares $C_{i}$ exceeds 36 , the method is successful (the bound of 36 is lowered to 16 in the following sections). This answers Lassak's question in the affirmative.

Example 2. Use the same curve $\varphi$ as in Example 1 to on-line cover the unit square with a sequence of rectangles $D_{i}$, each of which is "domino-like", i.e., similar to the two-by-one rectangle. A slight modification of the argument of Example 1 yields the conclusion that the method is successful if the total area of the rectangles $D_{i}$ exceeds 18 (this bound is lowered to 8 by Remark 3 at the end of Section 4).
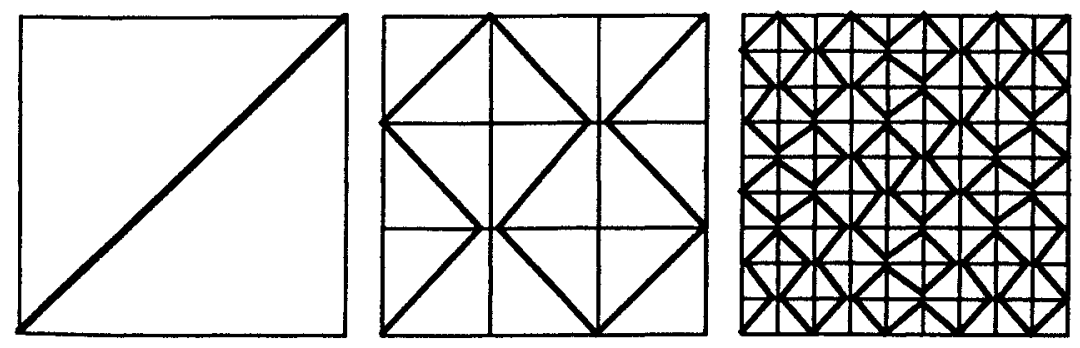

Fig. 2. Three initial approximations of $\varphi$. 
The factor of $9^{-1}$ associated with the curve $\varphi$ used in the above examples is crucial in estimating the efficiency of the resulting on-line covering method. Tentatively, we call this factor the efficiency coefficient of the curve. Obviously, a Peano curve with a greater efficiency coefficient would yield a better efficiency estimate for each of the above problems. The general construction of the next Section produces a Peano curve filling the $d$-dimensional unit cube, with an efficiency coefficient of $2^{-d}$. The precise definition of the efficiency coefficient and the problem of maximum efficiency of a Peano curve will be addressed elsewhere.

\section{An Efficient Cube-Filling Peano Curve}

Assume the following notation:

$I=[0,1]$ denotes the unit interval of real numbers.

$I^{d}=\left\{\left(x_{1}, x_{2}, \ldots, x_{d}\right) \in E^{d}: x_{i} \in I\right.$ for $\left.1 \leq i \leq d\right\}$ is the $d$-dimensional unit cube.

$I_{j, k}$ is the $j$ th subinterval of $I$ under the regular partition of $I$ into $2^{d k}$ subintervals,

$$
I_{j, k}=\left[\frac{j-1}{2^{d k}}, \frac{j}{2^{d k}}\right] \quad\left(j=1,2,3, \ldots, 2^{d k} ; k=1,2, \ldots\right)
$$

$P_{k}^{d}$ denotes the regular partition of $I^{d}$ into $2^{d k}$ congruent cubes, each of edge-length $2^{-k}(k=1,2, \ldots)$.

The ( $d$-dimensional) volume of a set $S \subset E^{d}$ is denoted by $|S|$. In particular, if $J$ is an interval, $|J|$ denotes the length of $J$.

The object of this section is to construct a continuous function $\varphi^{d}$ from $I$ onto $I^{d}$, a Peano curve filling the cube, satisfying the following efficiency conditions:

(3.1) The image $\varphi^{d}\left(I_{j . k}\right)$ is one of the cubes in the partition $P_{k}^{d}$, for each $k$ and each $j=1,2,3, \ldots, 2^{d k}$.

(3.2) For each $j=1,2,3, \ldots, 2^{\mathrm{d} k}-1$, the cubes $\varphi^{\mathrm{d}}\left(I_{j, k}\right)$ and $\varphi^{\mathrm{d}}\left(I_{j+1, k}\right)$ have a common facet.

The function $\varphi^{d}$ is defined as the limit of a uniformly convergent sequence of continuous, piecewise-linear approximations $\left\{f_{1}^{d}, f_{2}^{d}, \ldots\right\}$. The first term of the sequence, $f_{1}^{d}$, plays a crucial role as a "building block" of the repeating pattern in the construction of the subsequent terms $f_{i}^{d}$ (compare, for instance, [4], [5], or [7]). The functions $f_{1}^{d}(d=1,2, \ldots)$ are defined by induction with respect to $d$ as follows.

1. $f_{1}^{1}$ is defined by $f_{1}^{1}(x)=x$.

2. Assume $f_{1}^{d}$ has been defined so that, for each $j$, the subinterval $I_{j, 1}$ is mapped linearly onto an oriented edge of a cube in $P_{1}^{d}$, and that to each oriented edge 
$f_{1}^{d}\left(I_{j, 1}\right), j=1,2,3, \ldots, 2^{d}$, a cube in $P_{1}^{d}$ has been assigned in a one-to-one manner, containing the edge. We can think of $f_{1}^{d}$ as a path of length $2^{d}$, running through the (short) edges of the partition $P_{1}^{d}$ and "visiting" each of the $2^{d}$ small cubes of the partition. Observe that $f_{1}^{1}$ has this property. Using this approach, we can formally write

$$
f_{1}^{d}=\left\langle e_{1} e_{2} \cdots e_{2^{d}}\right\rangle
$$

where $e_{j}$ is an (oriented) edge of the partition $P_{1}^{d}$, the one on which $I_{j, 1}$ is mapped by $f_{1}^{d}$. Let $q_{j}$ be the small cube assigned to the edge $e_{i}$. For a description of the edges of $f_{1}^{d+1}$ and of the assignment of small cubes to them, let us assume that $I^{d} \subset I^{d+1}$ by identifying the point $\left(x_{1}, x_{2}, \ldots, x_{d}\right) \in E^{d}$ with the point $\left(x_{1}, x_{2}, \ldots, x_{d}, \frac{1}{2}\right) \in E^{d+1}$ and thinking of the $x_{d+1}$-axis as being vertical. Thus, each oriented edge of $P_{1}^{d}$ is an oriented edge of $P_{1}^{d+1}$, and each cube $q$ of $P_{1}^{d}$ is a facet of exactly two cubes of $P_{1}^{d+1}$ : one, $q^{0}$, lying below $q$, and the other one, $q^{1}$, lying above $q$. Now, define $f_{1}^{d+1}$ by augmenting the path $f_{1}^{d}$ with certain vertical edges of $P_{1}^{d+1}$. Namely, set

$$
f_{1}^{d+1}=\left\langle\uparrow e_{1} e_{2} \downarrow \uparrow e_{3} e_{4} \downarrow \uparrow \cdots \downarrow \uparrow e_{2^{d-1}} e_{2^{d}} \downarrow\right\rangle,
$$

where each vertical arrow denotes an oriented vertical edge in the lower half of $P_{1}^{d+1}$; each up-arrow $\uparrow$ terminates at the initial point of the following edge $e_{i}$, and each down-arrow $\downarrow$ starts at the terminal point of the preceding edge $e_{j}$.

Next, to a horizontal edge $e$ of $f_{1}^{d+1}$ assign the cube $q^{1}$ above the cube $q$ assigned to the very same edge in $P_{1}^{d}$, and to a vertical edge $\uparrow$ or $\downarrow$ of $f_{1}^{d+1}$ assign the cube $q^{0}$ below the cube $q$ assigned to the neighboring horizontal edge in $P_{1}^{d}$. In this fashion, each of the $2^{d+1}$ cubes of $P_{1}^{d+1}$ is assigned to exactly one of the oriented edges forming the path $f_{1}^{d+1}$, and each of them contains the edge to which it is assigned.

Figure 3 shows the paths $f_{1}^{2}$ and $f_{1}^{3}$ with the small cubes attached to the corresponding edges (the small cubes are shown pushed apart from each other for better visibility of the assignment).
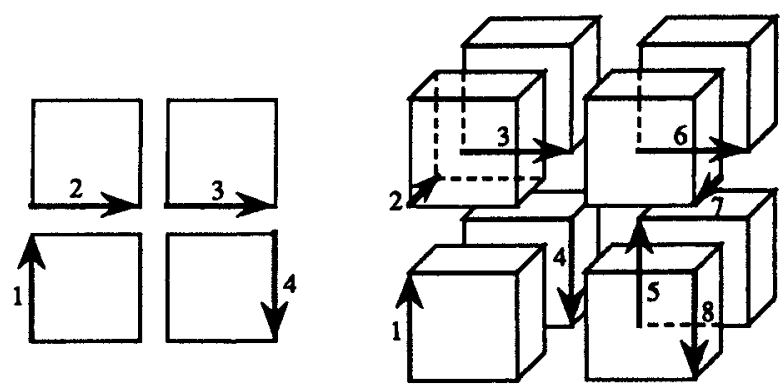

Fig. 3. The paths $f_{1}^{2}$ and $f_{1}^{3}$. 

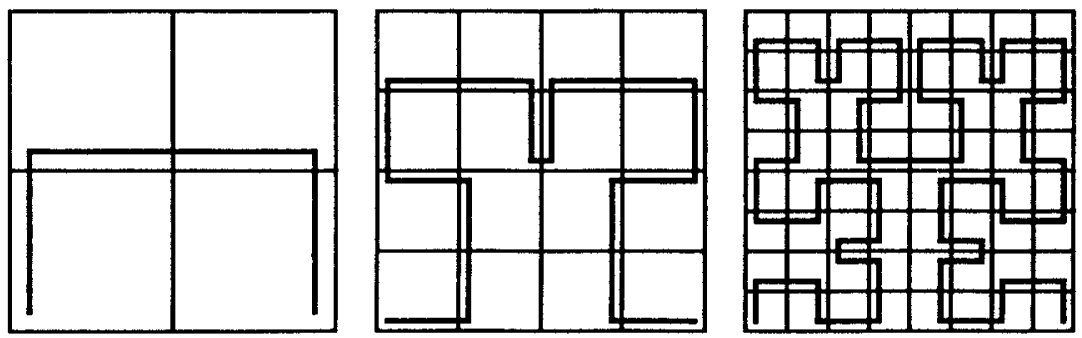

Fig. 4. The paths $f_{1}^{2}, f_{2}^{2}$, and $f_{3}^{2}$.

For a fixed $d$, the paths $f_{i}^{d}$ are defined by induction with respect to $i$. Since $f_{1}^{d}$ has already been defined, we proceed with the inductive step. Assume that $f_{i}^{d}$ has been defined as a path consisting of $2^{d i}$ oriented edges in $P_{i}^{d}$, and that to each edge $e$ of $f_{i}^{d}$ a (small) cube $q$ of $P_{i}^{d}$ has been assigned, containing the edge. Observe that the path $f_{1}^{d}$ starts at the origin $O=(0,0, \ldots, 0)$ and ends at $A=(1,0, \ldots, 0)$, for every $d$.

Now the path $f_{i+1}^{d}$ is obtained by replacing each edge $e$ of $f_{i}^{d}$ with a path running through the small cube $q$ assigned to $e$ in a way similar to the way $f_{1}^{d}$ runs through $I^{d}$. More precisely, a similitude between the cube $I^{d}$ and the cube $q$ exists which sends the oriented edge $\overrightarrow{O A}$ onto the oriented edge $e$; we replace $e$ with the image of $f_{1}^{d}$ under this similitude. The similitude determines not only the refinement of the edge $e$, but also the assignment of the smaller cubes (in $P_{i+1}^{d}$ ) to the shorter edges that replace $e$.

We should note here that, for $d \geq 3$, the similitude in question is not unique, as more than one isometry of the cube $I^{d}$ onto itself exists under which the oriented edge $\overrightarrow{O A}$ is invariant. However, for our purpose it does not matter which similitudes are chosen to refine the edges of $f_{i}^{d}$.

This completes the inductive definition of the sequence of functions $\left\{f_{i}^{d}\right\}$. Figure 4 shows the paths $f_{1}^{2}, f_{2}^{2}$, and $f_{3}^{2}$, slightly moved off the edges and into the assigned squares, to make the square assignment more visible.

The sequence of paths $\left\{f_{1}^{d}, f_{2}^{d}, \ldots\right\}$ converges uniformly to a continuous function $\varphi^{d}$ that maps $I$ onto $I^{d}$, the Peano curve we intended to construct. The efficiency conditions (3.1) and (3.2) are consequences of the following properties of the construction: For each $i \geq k, f_{i}^{d}\left(I_{j, k}\right)$ is contained in one cube of $P_{k}^{d}$, namely, in the cube of $P_{k}^{d}$ assigned to the edge $f_{k}^{d}\left(I_{j, k}\right)$, and the cubes of $P_{k}^{d}$ assigned to two consecutive edges of the path $f_{k}^{d}$ have a common facet.

\section{Proof of the Theorem}

According to (3.1), the image under $\varphi^{d}$ of every interval $I_{j, k}$ is a cube $q$ of $P_{k}^{d}$. Observe that $\left|I_{j, k}\right|=|q|=2^{-d k}$. Since every interval $J \subset I$ with $|J|=2^{-d k}$ is contained in the union of two adjacent intervals $I_{j, k}$ and $I_{j+1, k}$, it follows by (3.2) 
that:

(4.1) For every interval $J \subset I$ with $|J|=2^{-d k}$, the image $\varphi^{d}(J)$ is contained in a cube of volume $2^{d}|J|$.

Next, every interval $J \subset I$ is contained in an interval $J^{\prime} \subset I$ with $\left|J^{\prime}\right|=2^{-d k} \leq 2^{d}|J|$ for some $k=0,1,2, \ldots$ To see it, just let $k$ be the least interger for which $2^{-d(k+1)}<|J|$. Thus, by (4.1), we get:

(4.2) For every interval $J \subset I$, the image $\varphi(J)$ is contained in a cube $C$ with $|C| \leq 4^{d}|J|$.

Now we apply the on-line covering method described in Section 2. Suppose $\left\{C_{i}\right\}$ is a sequence of cubes in $E^{d}$ with $\sum_{i=1}^{\infty}\left|C_{i}\right|>4^{d}$ with which the unit cube $I^{d}$ is to be covered on-line. We may assume that $\left|C_{i}\right| \leq 1$ for each $i$. Place $C_{1}$ so that it covers $\varphi^{d}\left(\left[0, c_{1}\right]\right)$ where $c_{1}=4^{-d}\left|C_{1}\right|$. Statement (4.2) assures that it is possible to do so. Next, place $C_{2}$ so that it covers $\varphi^{d}\left(\left[c_{1}, c_{2}\right]\right)$ where

$$
c_{2}=\min \left\{1, c_{1}+4^{-d}\left|C_{2}\right|\right\}
$$

and proceed in this fashion. The procedure stops as soon as $c_{i}$ reaches the value of 1 , at which time $I^{d}$ is covered by a finite number of the $C_{i}$ 's.

Remark 1. If the sequence of cubes $\left\{C_{i}\right\}$ is finite, then the assumption

$$
\sum_{i=1}^{\infty}\left|C_{i}\right|>4^{d}
$$

can be relaxed to $\sum_{i=1}^{\infty}\left|C_{i}\right| \geq 4^{d}$.

Remark 2. Since each of the cubes of $P_{k}^{d}$ is homothetic to $I^{d}$, the theorem proved above can be modified, without any changes in the proof, as follows:

Let $\left\{C_{i}\right\}$ be a sequence of cubes each of which is homothetic to Id If $\sum_{i=1}^{\infty}\left|C_{i}\right|>4^{d}$, then the sequence admits a translational on-line covering of $I^{d}$.

Remark 3. For each of the two versions of the theorem, consider a special case, in which the sequence of cubes $\left\{C_{i}\right\}$ is self-repeating, i.e., the cubes $C_{2 i-1}$ and $C_{2 i}$ are congruent for $i=1,2, \ldots$. Then for the existence of a [translational] on-line covering of $I^{d}$ it is enough to assume that $\sum_{i=1}^{\infty}\left|C_{i}\right|>2^{d+1}$. This efficiency estimate is almost as good as the best one, $2^{d}-1$, for the usual covering problem, without the on-line restriction (the bound of $2^{d}-1$ could not be lowered there even if all cubes $C_{i}$ were congruent). If rotations are allowed, then the bound of $2^{d+1}$ holds even if the congruent cubes in each pair are glued to each other along a common facet (recall Example 2 of Section 2, the "domino-like" rectangles). 


\section{Acknowledgment}

Many thanks to Piotr Minc for his helpful comments and suggestions.

\section{References}

1. A. Bezdek and K. Bezdek, Eine hinreichende Bedingung für die Uberdeckung des Einheitswürfels durch homothetische Exemplare im $n$-dimensionalen Euklidischen Raum, Beiträge zur Algebra und Geometrie 17 (1984), 5-21.

2. H. Groemer, Covering and packing properties of bounded sequences of convex sets, Mathematika 29 (1982), 18-31.

3. $\mathrm{H}$. Groemer, Coverings and packings by sequences of convex sets, in Discrete Geometry and Convexity, Annals of the New York Academy of Sciences, Vol. 440, New York Academy of Science, New York, 1985, pp. 262-278.

4. J. G. Hocking and G. S. Young, Topology, Addison-Wesley, Reading, MA, 1961.

5. K. Kuratowski, Introduction to Set Theory and Topology, Pergamon Press, Oxford, 1972.

6. M. Lassak and J. Zhang, An on-line potato-sack theorem, Discrete \& Computational Geometry 6 (1991), 1-7.

7. J. R. Munkres, Topology, a First Course, Prentice-Hall, Englewood Cliffs, NJ, 1975.

Received December 7, 1992. 